\title{
Classification versus inference learning contrasted with real-world categories
}

\author{
Erin L. Jones • Brian H. Ross
}

Published online: 15 December 2010

(C) Psychonomic Society, Inc. 2010

\begin{abstract}
Categories are learned and used in a variety of ways, but the research focus has been on classification learning. Recent work contrasting classification with inference learning of categories found important later differences in category performance. However, theoretical accounts differ on whether this is due to an inherent difference between the tasks or to the implementation decisions. The inherent-difference explanation argues that inference learners focus on the internal structure of the categories - what each category is like-while classification learners focus on diagnostic information to predict category membership. In two experiments, using real-world categories and controlling for earlier methodological differences, inference learners learned more about what each category was like than did classification learners, as evidenced by higher performance on a novel classification test. These results suggest that there is an inherent difference between learning new categories by classifying an item versus inferring a feature.
\end{abstract}

Keywords Categorization · Category learning ·

Classification learning $\cdot$ Inference learning

Categories underlie a variety of cognitive tasks, including problem solving, classification, prediction, explanations, and inferences. Knowing the category an object belongs to enables access to categorical knowledge acquired from past

E. L. Jones $(\bowtie) \cdot$ B. H. Ross

Department of Psychology, University of Illinois,

603 E. Daniel St.,

Champaign, IL 61820, USA

e-mail: eljones3@illinois.edu experiences. We use category knowledge when retrieving the formula for solving permutation problems, classifying an approaching person as a soldier, or deciding whether to flee from an approaching Labrador retriever. One can argue that the importance of categories is not in knowing that the object in front of you is a chair or a table, but rather in using the category knowledge associated with the object to make inferences about it, such as whether to sit on it or place a glass on it (J. R. Anderson, 1991; Murphy, 2002). We learn categories in many ways, and it is likely that differences in processing during learning lead to differences in category knowledge (Markman \& Ross, 2003). The goal of the present study is to examine whether two principal ways of learning categories lead to critical differences in category knowledge.

Almost all empirical studies and extant theories have focused on one type of learning, classification learning, in which a learner determines what category an item belongs to. However, category learning is more than just classification, and it will be critical to understand these other ways of learning as well as the degree to which learners can flexibly use categories for a variety of tasks beyond classification.

\section{Classification versus inference learning: Paradigms and results}

To better understand how different ways of learning affect what knowledge is acquired, some recent studies have compared classification learning to another major means of category learning, inference, where a learner predicts a missing feature of a classified item (e.g., A. L. Anderson, Ross, \& Chin-Parker, 2002; Chin-Parker \& 
Ross, 2004; Yamauchi \& Markman, 1998). The force of this comparison is that the two types of category-learning tasks can be set up similarly, with the main difference being whether people predict a missing category label (classification) or a missing feature (inference). In the typical classification learning paradigm, learners are presented with an exemplar from one of two categories, asked to classify the exemplar, and then provided with feedback. For example, they might be shown some fictional creature and asked if it is a Deeger or Koozle. In the typical inference learning paradigm, learners are presented with an exemplar from one of two categories, along with its category label but without one of its features, asked to provide the missing feature's value, and then provided with feedback. Here, the fictional creature could be presented with a Deeger label but without its tail, and the learner would be given two tails to choose from. In both conditions, learning trials continue until the participant reaches a predetermined learning criterion or a fixed number of trials set by the experimenter.

Previous studies have generally used a family resemblance category structure (Rosch \& Mervis, 1975) with binary-valued features. Table 1 shows an illustrative family resemblance category structure with four binary dimensions. For each category, each dimension has a value that is prototypical, or common across category members, and atypical for the other category's members. For instance, if striped wings are prototypical for category A and an exemplar has striped wings, this would be signified by a 1 in the first position. To capture variation across category members, three of the four dimensions for an exemplar are

Table 1 Family resemblance category structure

\begin{tabular}{|c|c|c|c|c|c|}
\hline \multirow[t]{2}{*}{ Exemplars } & & \multicolumn{4}{|c|}{ Feature Dimensions } \\
\hline & & 1 & 2 & 3 & 4 \\
\hline \multirow[t]{5}{*}{ Category A } & Prototype & 1 & 1 & 1 & 1 \\
\hline & 1 & 1 & 1 & 1 & 0 \\
\hline & 2 & 1 & 1 & 0 & 1 \\
\hline & 3 & 1 & 0 & 1 & 1 \\
\hline & 4 & 0 & 1 & 1 & 1 \\
\hline \multirow[t]{5}{*}{ Category B } & Prototype & 0 & 0 & 0 & 0 \\
\hline & 1 & 0 & 0 & 0 & 1 \\
\hline & 2 & 0 & 0 & 1 & 0 \\
\hline & 3 & 0 & 1 & 0 & 0 \\
\hline & 4 & 1 & 0 & 0 & 0 \\
\hline
\end{tabular}

Note. Each dimension is a feature (e.g., wing type), and each feature has two values (e.g., striped wings and spotted wings), indicated by 1 and 0 set to its category's prototypical value, and one dimension, known as the exception feature, is set to the other category's prototypical value.

Although the paradigms look quite similar, a number of studies have shown that classification and inference learners perform differently on a variety of later category tasks (see Markman \& Ross, 2003, for a review). Classification learners focus on diagnostic features (i.e., those that distinguish between categories), whereas inference learners focus on features that are common to many category members, even if they are not diagnostic (ChinParker \& Ross, 2004). Classification learners are more sensitive to the training exemplars than are inference learners (A. L. Anderson et al., 2002; Yamauchi \& Markman, 1998), whereas inference learners more readily notice underlying relational commonalities than do classification learners (Erickson, Chin-Parker, \& Ross, 2005; Rehder \& Ross, 2001).

\section{Classification versus inference: Explanations}

If performance differences between classification and inference learning are due to inherent task differences, it suggests that different category-learning tasks may lead to differences in category knowledge. If so, then categorylearning theories based only on classification learning (almost all current theories) may not provide a strong foundation for a general understanding of category learning. Rehder, Colner, and Hoffman (2009) differentiated three explanations for the classification-inference performance differences (which range from inherent task differences to differences due to particulars of the paradigms): the category-centered learning hypothesis (e.g., Markman \& Ross, 2003; Yamauchi \& Markman, 1998), anticipatory learning (Rehder et al., 2009), and the set-of-rules model (Johansen \& Kruschke, 2005). We describe these three explanations below and then propose how they may be tested.

First, the category-centered learning hypothesis argues that inference learners, because they are given a classified item, are focused on within-category information that will predict missing features, thus leading learners to try to understand the internal structure of the category. Hence, they learn prototypical features (whether diagnostic or not) and the empirical and meaningful correlations among features. In contrast, classification learners are focused on features that provide the most diagnostic information - those that best predict the category label - consistent with many current classification models (e.g., Kruschke, 1992; Nosofsky, 1984; see Rehder \& Hoffman, 2005). Thus, this view argues for an inherent difference between inference and category learning that is likely, in many cases, to lead to important differences 
in category knowledge (for further implications, see our General Discussion consideration of real-world task differences). One can view the tasks as almost equivalent, in that the inference task requires predicting a feature and the classification task requires predicting a category label, but there is evidence that people treat labels and features quite differently. Category labels license more inferences (e.g., Gelman \& Markman, 1986) and are treated as more central and stable than features (Gelman \& Heyman, 1999). Also, when given a label, one is able to focus on that particular category and ignore other categories; when given features but no label, one must consider multiple categories. These findings suggest that the label-feature distinction is a critical difference between these tasks, resulting in differences in goals and strategies when learning categories.

Second, the anticipatory-learning account posits that inference learners will spread their attention to various feature dimensions because they anticipate being asked about those dimensions on future trials (Rehder et al., 2009). As a result, while classification learners focus only on learning a sufficient number of features that predict the label (because the label is the only thing that could be asked about on a classification trial), inference learners focus not just on features helping to infer the missing feature, but also on other features that may be asked about later. This is a plausible explanation given that, in all previous work comparing classification and inference, inference learners have been asked about multiple features while classification learners have only been asked to provide the category label. For example, on some trials, inference learners may be asked to choose which tail the presented Deeger has, and on other trials, which legs it has. Inference learners might be learning more about what each category is like because they are asked about multiple features and are preparing for future queries.

Two studies have addressed this issue, with conflicting results. A. L. Anderson et al. (2002) found that inference learners who only inferred two out of four features still learned more about the nonqueried features than did classification learners. However, Rehder et al. (2009) showed that when participants are explicitly told they will only be asked about some particular features, their looking time to nonqueried features decreases drastically (although the Rehder et al. features were not part of the usual integrated figures, but were spatially separated)

Third, the set-of-rules model proposes that, because of the peculiarities of the studies, inference learners simply learn associations between the category label and each feature (Johansen \& Kruschke, 2005). According to this account, the inference-classification performance differences can be explained by two differences in how category structures were implemented in previous studies. One, inference learners were not asked to infer exception features (e.g., the third dimension for Category A's exemplar 1101 in Table 1). Therefore, for inference learners in these studies, the category label was perfectly predictive of the feature value to be inferred, with all Category A inferences on the third dimension requiring the value 1 . However, such an association was not true for classification learners-no single feature perfectly predicted the category label across all trials. This difference between classification and inference could lead inference learners to focus more on the prototypical values of the category than would classification learners, who are more aware of the exception features during learning. Two, the association between the category label and features is higher than the association between a feature and other features within the same category. ${ }^{1}$ For example, when the category label is " $\mathrm{A}$," the first feature dimension is a " 1 " $75 \%$ of the time. However, when the first feature dimension is a " 1 ," the second feature dimension is a " 1 " only $50 \%$ of the time. This asymmetry in associations could cause the learner to treat the label differently from features and thus result in differences between the tasks.

In summary, the category-centered learning hypothesis proposes an inherent difference between the tasks, whereas the anticipatory-learning and set-of-rules accounts suggest that differences arise from how the task is implemented (although the anticipatory-learning account allows that these differences may also occur in real-world situations). These alternative explanations depend on two particular implementation decisions. First, classification learners infer the same category dimension (the category label) on every trial, whereas inference learners infer multiple dimensions (features). Second, the category structure leads the two tasks to have unequal associations between queried and presented features (as detailed in the preceding paragraph). In addition to these two implementation differences, there is a simple methodological difference that could provide an uninteresting explanation for the task differences: the type of information queried. In many of the studies showing classification-inference differences, classification learners were asked to provide a verbal label, while inference learners were asked to provide a visual feature. Learning about categories often includes learning about both visual information (what category members look like) and nonvisual information (e.g., what members eat, their ferocity). Being asked about visual information could cause learners to pay more attention to other visual information than if they were asked about verbal information. Additionally, it has been shown that visual information is remembered better than verbal information (e.g., Paivio, Rogers, \& Smythe, 1968; Shepard, 1967). Our experiments

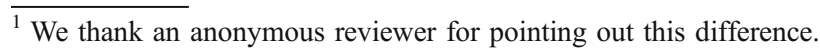


avoid this confound and also address the theoretically motivated implementation issues.

\section{The present experiments}

The goal of the present experiments is to contrast these explanations of classification-inference differences. Only the category-centered learning account proposes that inherent differences between inferring a label and inferring a missing feature (with a label provided) lead to the performance differences. To test these accounts, the methodological issues underlying the alternative explanations - number of queried dimensions, the implementation of the category structure, and the type of queried feature-were equated across the two tasks. Now the category-centered account still predicts that the groups will differ, whereas the anticipatory-learning and set-of-rules accounts could no longer explain any differences between classification and inference learners' performance at test.

First, we equated the amount of information queried during learning. Inference learners only inferred one feature, while classification learners only inferred the label. Second, to address the implementation of the category structure, we used two defining features (i.e., features that were $100 \%$ predictive of category membership, and thus perfectly correlated with the category label as well as with each other), one of which was chosen as the queried feature for the inference learners. Inference learners always received one defining feature, the category label, and a visual stimulus, and their task was to infer the other defining feature. Classification learners were provided with both defining features and a visual stimulus on each trial, and were asked to infer the category label. Third, the defining features were verbal predicates, so inference and classification learners were both asked questions about verbal information. Thus, both groups received the visual stimulus and two pieces of verbal information (either the two verbal predicates or one of them with the category label) and had to answer with the third piece of verbal information (the category label or the other verbal predicate respectively). For both classification and inference learners, using verbal information was sufficient for performing each task well, so attending to the visual stimulus was not necessary for either task.

One problem with making everything equivalent across these tasks is that participants may turn the inference task into a classification task if the stimuli are simple enough (e.g., by saying "Those are the triangle-eyed bugs and those are the square-eyed bugs"). We sought to reduce the chance of this occurring by using more complex, real-world bird categories (from Jacoby, Wahlheim, \& Coane, 2010), for which simple feature descriptions and category structures would be less obvious. An additional benefit of using these categories was that they reduced the arbitrariness of the labels. Participants may be more likely to treat bird labels as meaningful, knowing as they do that the labels correspond to instances in the real world. To our knowledge, only one other study (Sakamoto \& Love, in press) has used complex, real-world categories to compare classification and inference (though they did not equate the tasks in the ways done here).

The category-centered learning hypothesis proposes that inference learners are motivated to learn the internal structure of a category - what category members are like-while classification learners are motivated to learn diagnostic information for making a classification decision. If this hypothesis is correct, inference learners will be more likely to pay attention to and learn about information that is central to the category, even if it is not necessary for the inference task, whereas classification learners will focus only on information critical to the classification task. In our task, the visual stimulus (i.e., the bird pictures) provided visual information that was central to each category but unnecessary for both the classification and inference tasks. To test the differential predictions of the category-centered learning hypothesis, we examined what is often used as a central indication of category learning-classification of items that have not been seen before. Participants were given a novel classification test in which they had to classify new bird pictures (with verbal predicates omitted). Performance above chance on this test would indicate that learners acquired category-relevant information (visual features of the birds) that was an important aspect of the internal structure of the categories, even though learners in both tasks could have performed perfectly during learning without using this information (by relying only on verbal predicates). We hypothesized that if inference learning leads people to learn what each category is like, then inference learners would perform better than classification learners on tests that probed knowledge of what they learned about the visual characteristics of the bird categories, as evidenced by performance on the novel classification test.

\section{Experiment 1}

Experiment 1 investigated whether classification and inference learners develop different category representations for complex real-world categories. All participants learned about six different categories of birds (see Fig. 1). During learning, participants were provided with two verbal predicates and a picture of a bird from one of the categories. Inference learners were shown one defining-feature verbal predicate, the bird's picture, and the category label and were asked to provide the value of the other defining 


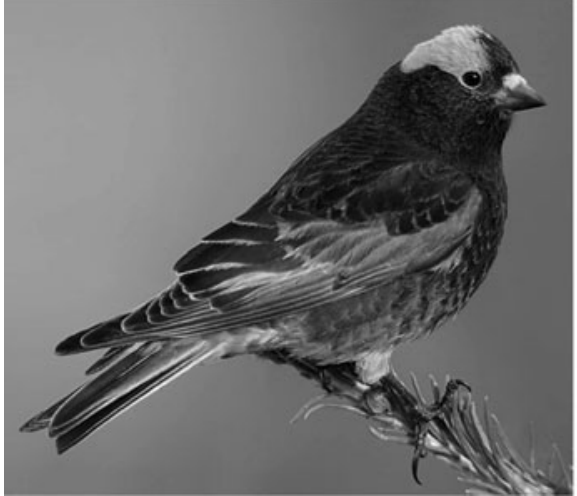

Finch

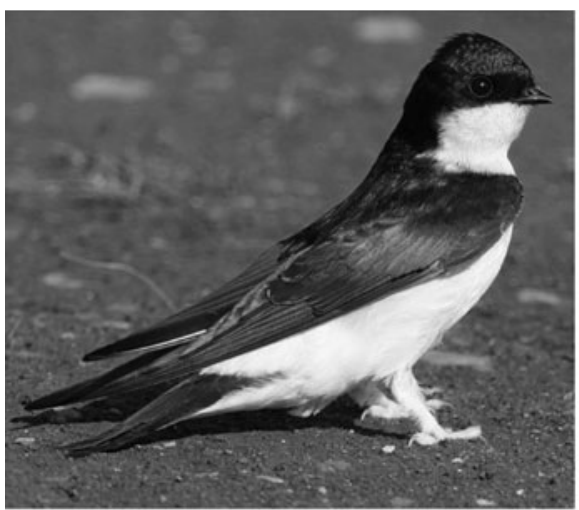

Swallow

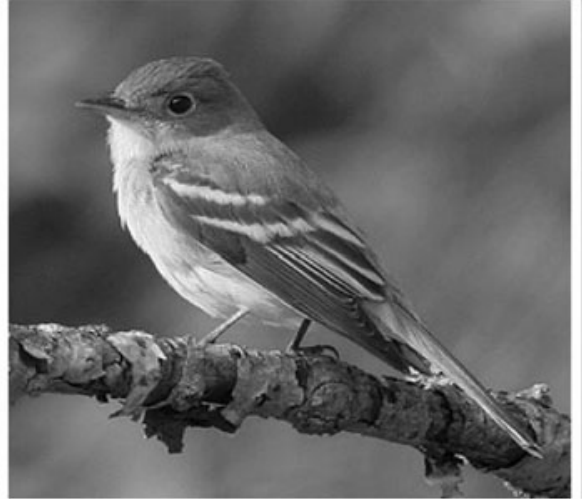

Flycatcher

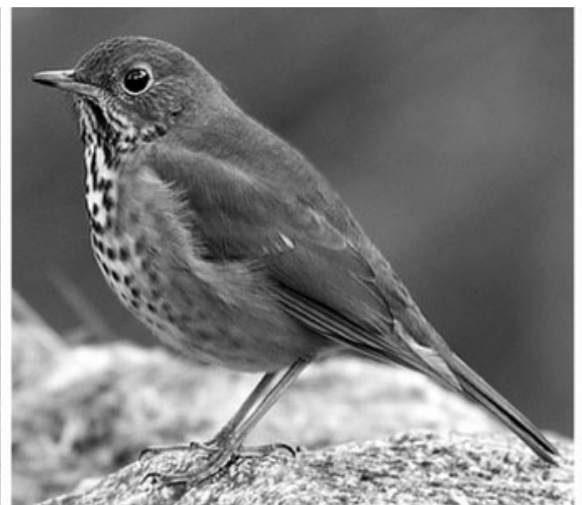

Thrush

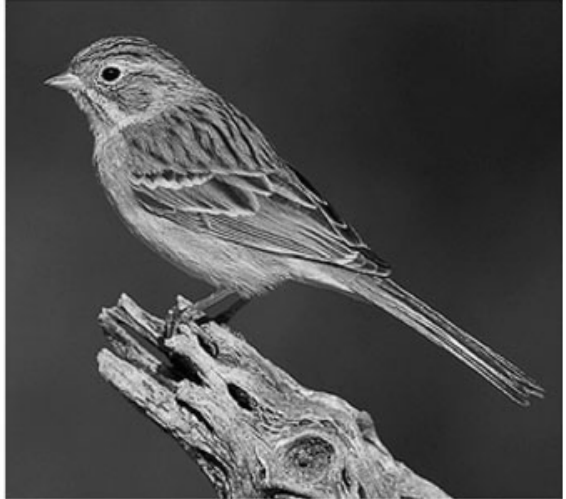

Sparrow

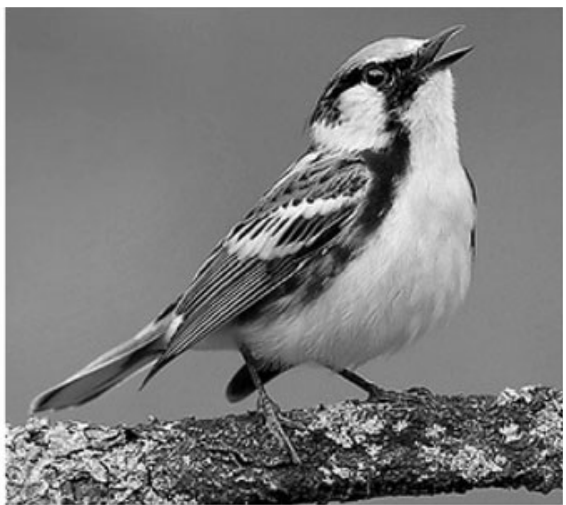

Warbler

Fig. 1 Example bird stimuli (pictures were in color)

feature on each trial. Classification learners were shown both defining-feature verbal predicates and the bird's picture and were asked to provide the category label on each trial. Both groups received feedback after their responses and learned for the same amount of time. Thus, during learning, both groups saw two pieces of perfectly correlated verbal information (either one verbal predicate and the category label or two verbal predicates) and a picture, and were asked to respond with the other perfectly correlated verbal information (either the other verbal predicate or the category label).

At test, we examined whether the two groups had important differences in categorical information. We chose the most common means of assessing category knowledge-classification of novel members of the categories (i.e., ones not seen during learning). For this test, verbal information was omitted from the trials, and learners had to use what they had learned about the visual properties of each bird category to make classification decisions. We predicted that inference learners would classify novel members of the bird categories better than classification learners, even though it is a classification test, because they would have attended more to what category members are like, including information not necessary for the learning task.
That is, even though the category label and the defining verbal features were all the information necessary to perform the classification or inference tasks during learning, we expected that the inference learners would learn more about the different families of birds.

We included two additional tests of participants' knowledge. One was a recognition test with classification (on each trial, participants determined whether the presented item was seen earlier in the experiment or not, and then decided which category it belonged to), to determine if any difference between classification and inference is partially due to a difference in memory for the specific items. For example, perhaps both groups would examine the pictures equally well, but the classification learners would focus more on the exact exemplars shown during study. If both types of learners attended to the visual information, but classification learners encoded specific exemplars while inference learners generalized across exemplars, then classification learners might show higher recognition memory performance. As the second test of knowledge, we included classification and inference tests of the verbal predicates (one at a time, without the bird pictures), to examine whether verbal information was learned equally well in both conditions. However, the main issue in the 
experiment was how the two groups would perform on the novel classification test-classifying items they had not seen before when no verbal information was presented. If inference learners attended more to the visual information to learn about what each category was like, beyond what was necessary during learning, then inference learners would show higher novel classification performance.

\section{Method}

\section{Design}

Participants were randomly assigned to one of two between-subjects conditions: classification or inference learning. Assignments of verbal feature values to categories, as well as the sets of response options, ${ }^{2}$ were counterbalanced across participants, resulting in eight counterbalancing groups.

\section{Participants}

Participants were 24 undergraduate students from the University of Illinois who participated for course credit.

\section{Materials}

The materials were color images of birds from various families in the Passeriformes order, compiled by Jacoby et al. (2010) from www.whatbird.com. The six bird families were finches, flycatchers, sparrows, swallows, thrushes, and warblers. For each family, 15 exemplars were used throughout the experiment: 6 exemplars in the study phase, 6 in the novel classification test, and 3 in the recognition test. The pictures during study and the novel classification test had the birds in a context (e.g., on a post, in a tree) to make clear that the task involved learning real-world categories. However, the recognition test pictures all showed birds with just a white background, to ensure that the context did not influence the judgments. To keep the groups of families equated for difficulty, the assignment of exemplars to each phase of the experiment was determined based on previous test performance data.

The verbal predicates were the nest material each bird uses and the food each bird eats. There were six values for each of these two attributes. For nest material, the values were grass, leaves, moss, mud, silk, and sticks. Each verbal predicate had the same structure: "This bird's nest is made of . For the food each bird eats, the values were berries, insects, nuts, plants, seeds, and worms. The structure of this verbal predicate was

\footnotetext{
$\overline{2}$ To make the task easier during learning, only three answers appeared at the same time, counterbalanced across participants.
}

"This bird eats . Values were chosen based on what real birds use to make their nests and what they actually eat, but the assignment of values to bird families was counterbalanced across participants, so they did not actually reflect what each of these bird families build their nests with and what they eat. Each verbal predicate was perfectly predictive of category membership.

\section{Procedure}

After giving informed consent, participants rated their knowledge about each bird family on a scale of $1-5$, to ensure that none of the participants already had knowledge about the birds. Next, participants were told that they would be learning about different bird families and would be tested on their knowledge of the families later.

First, participants completed a learning phase. For both classification and inference learning conditions, there were two blocks of 36 trials. There were six exemplars from each of the six bird families, presented in a random order for each of the two blocks.

Classification learners were shown a picture along with two verbal predicates (what the bird eats and its nest material). Figure 2 shows an example learning trial. The three pieces of information always appeared on the left half of the screen. During the learning phase, participants were presented with pieces of information in the following order (with each piece remaining on the screen until the end of the trial): Verbal Predicate 1, bird picture, and Verbal Predicate 2. For each new piece of information, there was a 3 -s delay until the next piece was shown. The two verbal predicates were equally likely to be displayed as Verbal Predicate 1 or 2 . Additionally, verbal predicates were equally likely to be displayed above or below the bird picture. After all the information was displayed, the question "What kind of bird is this?" and three possible answers were displayed on the right side of the screen. Even though there were six bird families, only three possible answers were displayed, to make the task easier. We grouped bird families into two sets of three, and their category labels were shown in these same sets throughout the learning phase. Participants clicked on their choice using the mouse, and there was no time limit to make a selection. Next, participants were given feedback (3s) as to whether they were correct or incorrect, and the statement "This is a [Correct bird family]" was displayed.

Inference learners' learning trials were the same as the classification learners', with one exception. Instead of a second verbal feature, these participants saw the statement "This is a [Correct bird family]." Figure 3 shows an example learning trial. All inference learners were given the nest material verbal predicate and were asked to infer what the bird eats ("What does this bird eat?" appeared along with 


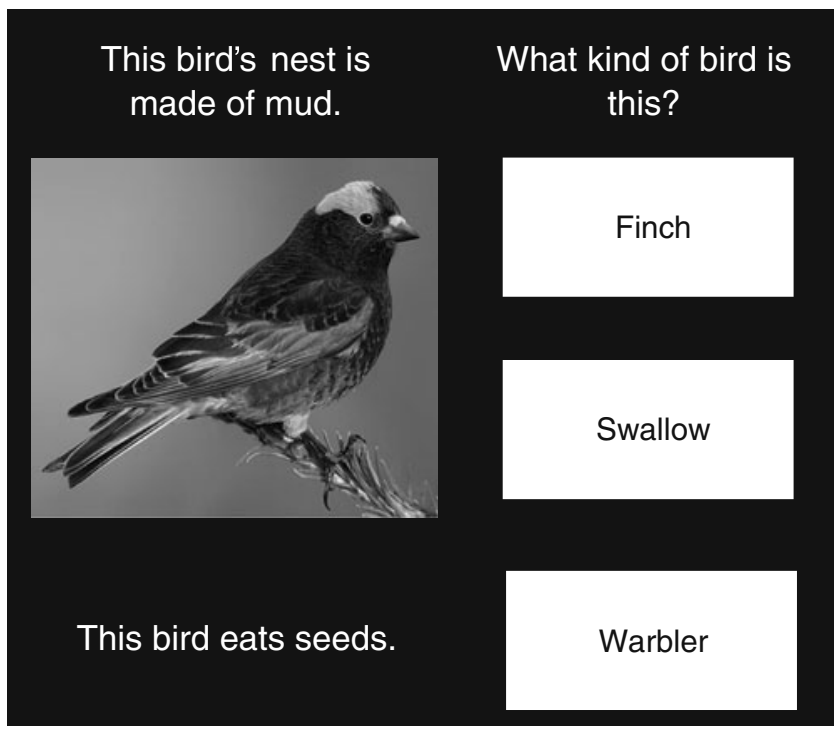

Fig. 2 Example classification learning trial. Information on the left appeared on the screen one piece at a time, until everything was displayed. The verbal predicates were equally likely to appear in either location on the screen

three possible answers, split into the same two groups of three as in the classification condition; these groupings were again kept throughout learning). The nest material predicate and the category label were equally likely to be presented before or after the bird picture (i.e., were equally likely to be the first or third piece of information presented).

All participants received the same four tests in the same order. First, participants completed a novel classification test. For each trial, participants viewed an exemplar and chose one of the six family names presented on the screen by clicking on its respective box with the mouse. Next, participants made confidence judgments about their response ["Likelihood of classification (16\%-100\%)"] using the number pad. There was no time limit on either the classification decision or the confidence judgment. There were 36 trials ( 6 for each bird family), presented in a random order for each participant.

Next, participants received a verbal predicate classification test. On each trial, participants were presented with one of the studied verbal predicates and were asked to click on which of the six bird families it was most likely to be true for. There were 12 verbal predicates ( 6 food and 6 nest material), which were presented in a random order for each participant.

Next, participants completed an old/new recognition test with classification. There were three types of exemplars: old exemplars (those presented in the study phase), new exemplars from previously studied categories, and new exemplars from categories never studied in the experiment (from other Passeriformes families: orioles, jays, grosbeaks, vireos, chickadees, and thrashers). There were 36 old exemplars, 18 new exemplars from old categories, and 18 new exemplars from new categories. Participants were presented with one bird picture at a time, and the task was to decide whether the presented bird was old or new by clicking the box on the screen that corresponded to their answer. After making the old/new decision, participants classified each bird into one of seven categories. The categories were each of the six families that had been learned about, as well as an "Other" category.

Finally, participants received a verbal predicate inference test. For this test, there were two types of questions asked: "What would a [Bird family] eat?" and "What kind of nest would a [Bird family] have?" The answers displayed were the six values that corresponded with the question being asked. Both questions were asked about each of the bird families, resulting in 12 trials, which were presented in a random order.

Afterward, participants completed a questionnaire that asked for each category: "What information did you use to determine if the bird was a ?" They were also asked to provide any additional information that aided in their decisions. The analyses of these data did not prove fruitful and will not be reported. Finally, participants were debriefed and thanked for their time.

\section{Results and discussion}

\section{Learning}

The two groups did not differ in learning performance on the queried information (feature or label). In the first block

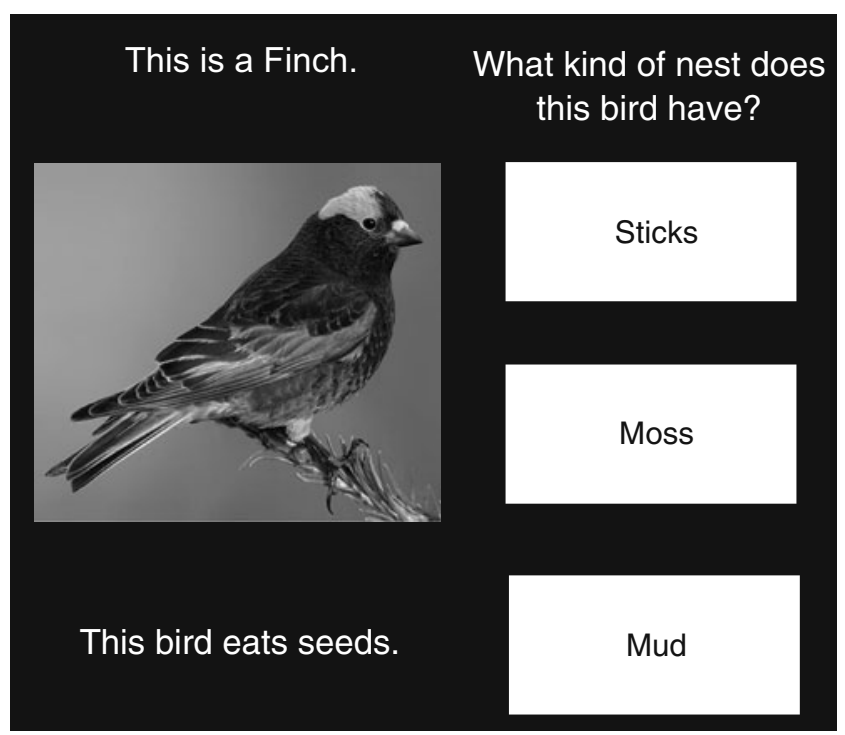

Fig. 3 Example inference learning trial. Information on the left appeared on the screen one piece at a time, until everything was displayed. The verbal predicates were equally likely to appear in either location on the screen 
of learning, classification learners $(M=.75, S D=.16)$ and inference learners $(M=.78, S D=.14)$ had similar performance, $t(22)<1$. In the final block of learning, both classification $(M=.95, S D=.08)$ and inference $(M=.94$, $S D=.09)$ learners performed equally well, $t(22)<1$. Learners in both conditions clearly learned about each category by the end of the learning phase.

\section{Tests}

Novel classification The critical result was performance on novel classification. Verbal information was omitted from these trials, so learners had to base their classification decisions on the bird pictures. It had not been necessary for either classification or inference learners to pay attention to the bird pictures during learning, so performance above chance would suggest that they went beyond learning what was critical for the learning task in order to learn what each category was like.

As predicted, inference learners had significantly higher accuracy on the novel classification test $(M=.26, S D=.05)$ than did classification learners $(M=.19, S D=.08)$, $t(22)=2.63, p<.05$. Inference learners' performance was higher than chance (which was .167), $t(11)=6.14$, $p<.001$, while classification learners' performance was not different from chance, $t(11)<1$. Inference learners were able to generalize what they had learned about the visual properties common across members of a bird category to novel birds at test, while classification learners failed to demonstrate any knowledge of the visual category information.

After each novel classification, participants gave a confidence rating for their response. One participant in the inference condition failed to provide confidence ratings and so is not included in this analysis. Inference learners' confidence ratings, regardless of whether the response was correct or incorrect, ${ }^{3}(M=.34, S D=.14)$ were higher than classification learners' $(M=.22, S D=.07), t(21)=2.67$, $p<.05$. $^{4}$

Recognition memory One explanation for the difference in novel classification performance is that the inherent difference between classification and inference is how learners encode exemplars during learning. Jacoby et al. (2010) suggested that there is a relationship between recognition memory and classification performance, and differences in this relationship across conditions may

\footnotetext{
${ }^{3}$ The confidence is presented for all responses because the proportions of correct responses are low and different in the two conditions. However, if one looks only at correct responses, inference learners' confidence ratings $(M=.39, S D=.18)$ were still higher than classification learners' $(M=.22, S D=.06), t(21)=2.99, p<.01$.

${ }^{4}$ This difference, however, was not replicated in Experiment 2, likely due to the addition of catch trials (see the Exp. 2 Method).
}

suggest differences in strategies. While inference learners focus more on what a category is like, perhaps classification learners focus more on individual exemplars. The recognition memory test was included to see if classification learning encouraged an exemplar-based approach to learning about the pictures. If this was the case, classification learners should have had better recognition memory performance (i.e., did you see this exact bird during learning?) than inference learners. ${ }^{5}$

For the recognition memory test, there were three types of trials: old items (36 items), new items from studied categories (18 items), and new items from unstudied categories (18 items). Table 2 shows recognition memory performance for each type of item by learning condition. A separate $d^{\prime}$ was calculated for each participant, and $d$ 's were then averaged by condition. There was no advantage for classification learners; performance was slightly better for the inference learners. For only studied category items (both old and new), there was no reliable difference in $d^{\prime}$ between inference learners $(M=0.81$, $S D=0.57)$ and classification learners $(M=0.51, S D=0.36)$, $t(22)=1.55, p>.05$. If items from nonstudied categories were included, there was still no reliable difference in $d^{\prime}$ between inference learners $(M=1.00, S D=0.49)$ and classification learners $(M=0.61, S D=0.39), t(22)=1.77, p$ $>.05$. Thus, there is no evidence that either task promoted better recognition memory than the other.

After participants decided that a bird was old or new, they classified it using either one of the six categories of birds or "Other." Table 3 shows classification performance for each type of item by learning condition: old items correctly identified as old in the recognition memory test, old items mistakenly identified as new, new items (broken down by studied categories and unstudied categories) correctly identified as new, and new items mistakenly identified as old. For birds that participants had correctly classified as old, inference learners were significantly more accurate than classification learners, $t(22)=2.23, p<.05$. This is further evidence that inference learners paid more attention to the bird pictures during learning than did classification learners. None of the other comparisons showed any clear difference (in all cases, $t(22)<1$ ).

Verbal predicate tests The verbal predicate classification test and verbal predicate inference test were included to ensure that both groups had learned about the verbal predicates, and additionally to see if learners were encoding

\footnotetext{
${ }^{5}$ It is possible that the change from a real-world background to a white background for test could hurt memory for exemplars, but we felt it critical to examine what participants had learned about the specific birds, not just the often distinctive backgrounds. We did consider using the white-background pictures for study as well, but decided it was more important to make the study pictures look as if they were of real-world items photographed in their contexts.
} 
Table 2 Experiment 1 recognition memory performance: Means and standard deviations by item type and condition

Item Type

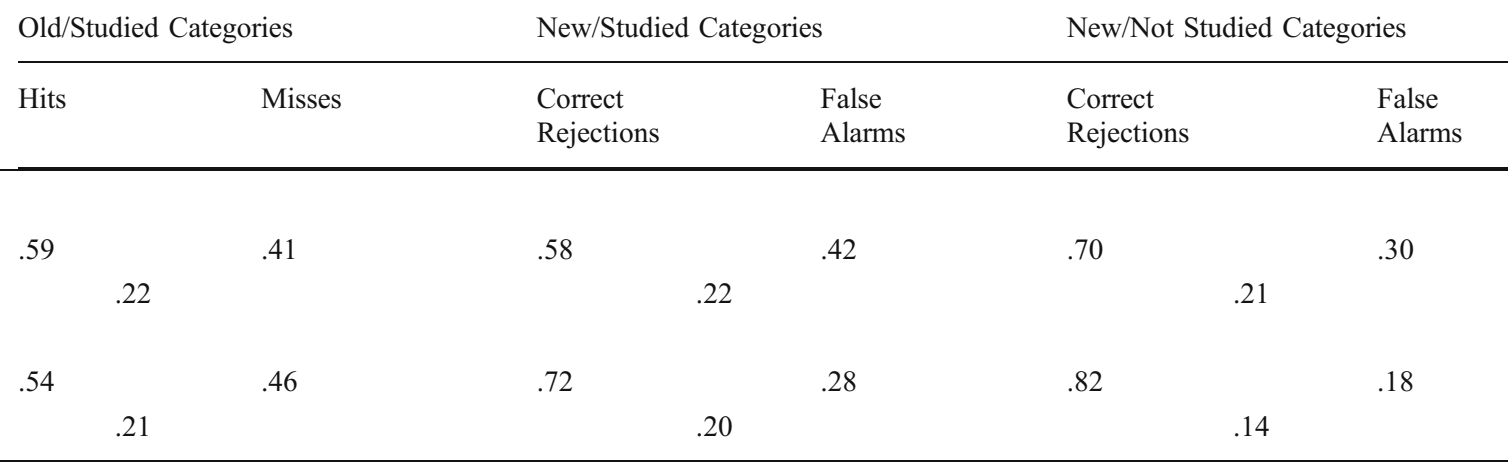

these features as a function of how they were queried during learning. For the verbal predicate classification test (where chance was .167), participants had to choose the name of the bird that most likely went with the presented statement. For this test, accuracy was similar for classification learners $(M=.69, S D=.16)$ and inference learners $(M=.67, S D=.26), t(22)<1$. For the verbal predicate inference test (where chance was .167), participants had to choose the feature value that was most likely true for the bird name presented. For this test, performance was also similar for classification learners $(M=.72, S D=.19)$ and inference learners $(M=.66, S D=.27), t(22)<1$. Both types of learners were able to learn about the verbal predicates and express what they knew equally well in both test formats.

\section{Summary}

During the learning phase, we equated the classification and inference tasks, except for the type of information asked about during learning - category label or feature. This study difference led to later performance differences between the two conditions. The critical result was that inference learners showed better performance than classification learners on a novel classification test. This result supports the categorycentered learning hypothesis. Inference learners focus on what each category is like, allowing them to generalize to novel exemplars better than classification learners. Classification learners, on the other hand, focus primarily on information that is diagnostic for category membership.

\section{Experiment 2}

It is important to examine different means of category learning and to understand how they each influence the representation of category knowledge. In Experiment 1, we were able to test the prediction made by the category-centered learning hypothesis, that the critical difference between the classification and inference tasks is whether a label or a feature is

Table 3 Experiment 1 postrecognition classification accuracy: Means and standard deviations by item type, participants' recognition memory response, and condition

Item Type

\begin{tabular}{|c|c|c|c|c|c|c|}
\hline & \multicolumn{2}{|c|}{ Old/Studied Categories } & \multicolumn{2}{|c|}{ New/Studied Categories } & \multicolumn{2}{|c|}{ New/Not Studied Categories } \\
\hline & Old & New & Old & New & Old & New \\
\hline \multicolumn{7}{|l|}{ Classification } \\
\hline Mean & .20 & .08 & .27 & .08 & $.06^{*}$ & $.74 *$ \\
\hline$S D$ & .11 & .11 & .17 & .10 & .15 & .35 \\
\hline \# Observations & 255 & 177 & 90 & 126 & 64 & 152 \\
\hline \multicolumn{7}{|l|}{ Inference } \\
\hline Mean & .31 & .10 & .27 & .08 & $.05^{*}$ & $.71^{*}$ \\
\hline$S D$ & .15 & .06 & .19 & .10 & .09 & .23 \\
\hline \# Observations & 234 & 198 & 60 & 156 & 39 & 177 \\
\hline
\end{tabular}

*Proportion of trials correctly classified as "Other" 
being asked about, and found that the two learning conditions led to differential performance on the critical novel classification test. Given the novelty and importance of this finding, it is essential for us to replicate the result. In addition, we attempted to increase both groups' examination of the bird pictures with longer study times and the introduction of catch trials in which not all the verbal information was presented.

\section{Method}

\section{Design}

Participants were randomly assigned to one of two betweensubjects conditions: classification and inference. There were three counterbalancing variables: assignment of verbal features to categories, response options, and (for inference learners) which of the two verbal predicates was inferred (nest material or what the bird eats). These were counterbalanced across participants, resulting in 16 counterbalancing groups.

\section{Participants}

In total, 32 undergraduate students from the University of Illinois participated for course credit. One participant was removed from the analysis for having prior knowledge of bird families.

\section{Materials}

The materials were the same as those used in Experiment 1.

\section{Procedure}

There were only two minor changes to the Experiment 1 procedure. For the learning phase, we increased the delay between the display of one piece of information and the next from 3 to $5 \mathrm{~s}$. Additionally, for 12 of the 36 trials in each block, one piece of verbal information was not displayed (half of the time, one kind of statement was omitted, and the other half of the time, the other kind of statement was). For these trials, the picture comprised half of the presented information. We hoped that this would lead to more attention to the pictures across both groups.

Results and discussion

\section{Learning}

As in Experiment 1, the two groups' learning performance did not differ. In the first block of learning, classification learners $(M=.71, S D=.09)$ and inference learners $(M=.71, S D=.12)$ had similar performance, $t(29)<1$. In the final block of learning, both classification learners $(M=.92, S D=.10)$ and inference learners $(M=.90, S D=.17)$ performed equally well, $t(29)<1$.

\section{Tests}

Novel classification We replicated the critical result from Experiment 1, that inference learners showed higher novel classification accuracy. The addition of the catch trials did not appear to have much influence on this performance. Inference learners had significantly higher performance on the novel classification test $(M=.28, S D=.09)$ than did classification learners $(M=.19, S D=.09), t(29)=2.55, p<.05$. Inference learners performed above chance (which was .167), $t(15)=$ 4.86, $p<.001$, but classification learners did not, $t(14)=$ $1.14, p>.05$. Inference learners paid more attention to the bird pictures during learning than did classification learners, and so were able to generalize to novel birds what they had learned about the different birds' visual properties.

Inference learners' confidence ratings, regardless of response accuracy ${ }^{6}(M=.30, S D=.11)$, were similar to the classification learners' ratings $(M=.31, S D=.12)$, $t(29)<1$, unlike in Experiment 1 (where the inference learners were more confident).

Recognition memory If classification learners are using an exemplar-based approach to learning, then their recognition memory performance should be better than inference learners' performance. Table 4 shows recognition memory performance for each item type by learning condition. A separate $d^{\prime}$ was calculated for each participant, and $d$ 's were then averaged by condition. For only studied category items (both old and new), there was no reliable difference in $d^{\prime}$ between inference learners $(M=0.53, S D=0.52)$ and classification learners $(M=0.58, S D=0.46), t(29)<1$. If items from nonstudied categories were included, there was still no reliable difference in $d^{\prime}$ between inference learners $(M=0.78, S D=0.44)$ and classification learners $(M=0.76, S D=0.41), t(29)<1$. There is no evidence that one learning task encouraged an exemplar-based encoding strategy more than the other.

After participants decided that a bird was old or new, they classified it using either one of the six categories of birds or "Other." Table 5 shows classification performance for each type of item by learning condition: old items correctly identified as old, old items mistakenly identified as new, new items (broken down by studied and unstudied categories) correctly identified as new, and new items mistakenly identified as old. There were no differences between classification and inference learners' performance $[t(29)<1$ for all comparisons, with the exception of birds that were new and from unstudied categories and that participants correctly classified as new, $t(29)=1.19$, $p>.05]$.

\footnotetext{
${ }^{6}$ If one looks only at correct responses, inference learners' confidence ratings $(M=.36, S D=.16)$ were still similar to the classification learners' $(M=.36, S D=.13), t(29)<1$.
} 
Table 4 Experiment 2 recognition memory performance: Means and standard deviations by item type and condition

Item Type

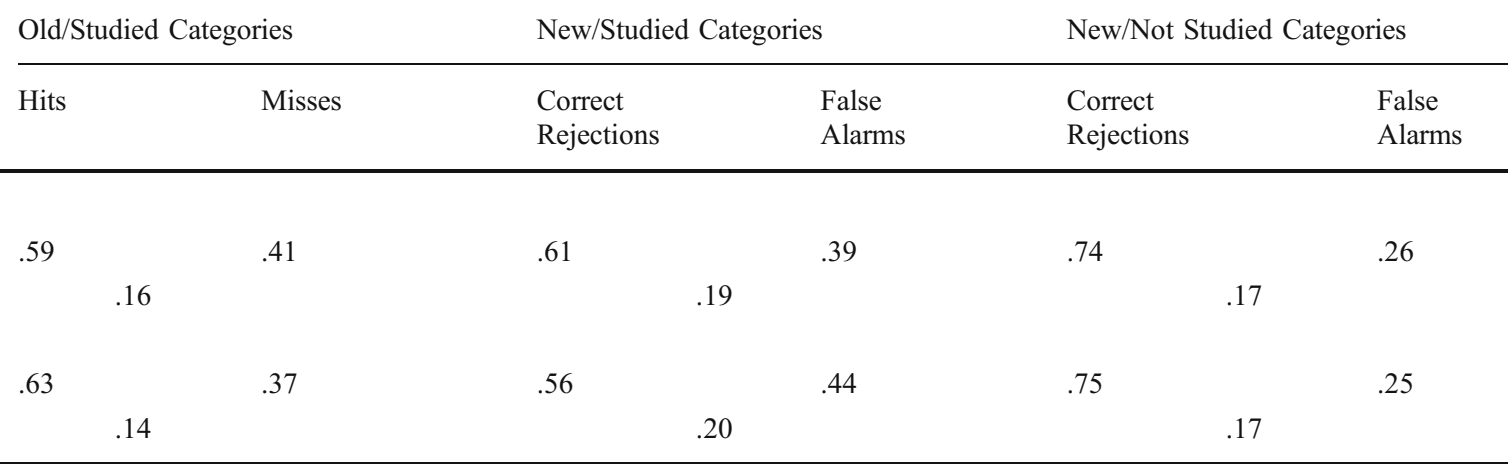

Verbal predicate tests As in Experiment 1, these tests were included to ensure that participants had learned the predicates and to see if classification and inference learners encoded them differently. Both types of learners performed equally well on both tests (where chance level for both tests was .167). For the verbal predicate classification test, performance was similar for classification learners $(M=.81, S D=.26)$ and inference learners $(M=.79, S D=.23), t(29)<1$. For the verbal predicate inference test, performance was also similar for classification learners $(M=.84, S D=.25)$ and inference learners $(M=.82, S D=.22), t(29)<1$.

\section{Summary}

We successfully replicated the main result from Experiment 1: Inference learners performed significantly above chance on the novel classification test, whereas the classification learners did not. This result supports the category-centered learning hypothesis, that learners develop strategies indicative of each task's goal. Inference learners' goal is to learn what each category is like, while classification learners' goal is to learn the information that best predicts category membership.

\section{General discussion}

Categories are useful for a broad range of tasks and are learned in a wide variety of ways, yet the relationship between use and learning has not been widely studied. Almost all of the experimental and theoretical work has focused on classification learning. Our experiments were designed to contrast performance from learning based on classification and another essential category use, inference. Do performance differences between classification and inference learning require an explanation such as the one offered by the category-centered learning hypothesis, which proposes an inherent difference between what is learned from the two tasks, or might the differences be explainable by implementation issues, such as the ones proposed by the

Table 5 Experiment 2 postrecognition classification accuracy: Means and standard deviations by item type, participants' recognition memory response, and condition

Item Type

\begin{tabular}{|c|c|c|c|c|c|c|}
\hline & \multicolumn{2}{|c|}{ Old/Studied Categories } & \multicolumn{2}{|c|}{ New/Studied Categories } & \multicolumn{2}{|c|}{ New/Not Studied Categories } \\
\hline & Old & New & Old & New & Old & New \\
\hline \multicolumn{7}{|l|}{ Classification } \\
\hline Mean & .21 & .13 & .20 & .10 & $.06^{*}$ & $.51 *$ \\
\hline$S D$ & .10 & .13 & .26 & .08 & .16 & .30 \\
\hline \# Observations & 316 & 224 & 105 & 165 & 69 & 201 \\
\hline \multicolumn{7}{|l|}{ Inference } \\
\hline Mean & .23 & .13 & .25 & .10 & $.04 *$ & $.64 *$ \\
\hline$S D$ & .14 & .12 & .20 & .09 & .07 & .31 \\
\hline \# Observations & 361 & 215 & 126 & 162 & 72 & 216 \\
\hline
\end{tabular}

*Proportion of trials correctly classified as "Other." 
anticipatory-learning account and the set-of-rules model? We eliminated three common differences between how these tasks have been implemented: number of queried dimensions, implementation of category structure, and type of queried feature. For the first time, the only differences between the two learning tasks were those that define the contrast between the classification and inference learning: the type of information queried (label or feature) and whether the provided verbal predicate was a feature or a label.

Across two experiments, we still found performance differences between classification and inference learners, providing support for the category-centered learning hypothesis and disconfirming the anticipatory-learning and set-of-rules explanations. Additionally, to our knowledge, this is the first time that inference learners outperformed classification learners on a classification test, therefore ruling out any simple idea that the test differences were due to transfer of processing from study. We address an alternative explanation for the results below, and then turn to larger issues about classification-inference differences.

\section{An alternative explanation}

A different explanation for these results is that both classification and inference learners "learned what they saw." Inference learners were presented with the label and bird picture throughout learning, while classification learners only saw the label and the bird picture on the same screen during feedback. By this account, both inference and classification learners were learning associations between whatever verbal predicates were on the screen and the bird pictures. If this explanation is true, then the novel classification test benefited inference learners because it tested for the association that they saw on the screen during study (the picture label), not because they learned better what category members were like.

There are many pieces of evidence to suggest that the "learn what you see" explanation is not plausible. First, this view predicts that inference learners should perform better on the verbal predicate tests (for trials involving the noninferred feature ${ }^{8}$ ). The reason is that these tests probed learners' label-feature association knowledge: Classification learners only saw the label with the features at feedback, whereas inference learners always saw the label and the noninferred feature together during the initial presentation of the information. Contrary to this prediction, classification learners had slightly higher performance than inference learners on both the verbal predicate classification and verbal predicate inference tests in both experiments

\footnotetext{
${ }^{7}$ We thank the reviewers for pointing out this possibility.

${ }^{8}$ For classification learners, both features were considered noninferred and were compared with the inference learners' noninferred feature performance.
}

(.07 to .12 advantages). Second, for inference learners this view predicts a benefit in the verbal predicate tests for the features not inferred during learning, relative to those that were inferred, since inference learners only saw the inferred feature paired with the label during feedback. However, inference learners were more accurate in providing the inferred feature across both experiments (.13 and .11, although this difference was only reliable for Exp. 2). ${ }^{9}$ Third, in Experiment 2, the number of trials for inference learners that included the picture-label association was reduced (due to the omission of one of the verbal predicates on $1 / 3$ of the trials). Despite fewer picture-label study opportunities, inference learners' novel classification performance did not decrease relative to Experiment 1 (it actually improved by $2 \%$ ). Fourth, earlier work that did not have the defining features or real-world materials showed an advantage for classification learners on classification tests (e.g., A. L. Anderson et al., 2002; Yamauchi \& Markman, 1998) when the category label had been shown during learning to the inference learners, suggesting that such tasks usually do not lead to the inference group advantage on a classification test seen here. Finally, this alternative explanation is contrary to the predictions of a well-supported view, transfer-appropriate processing theory, which has also been suggested as a framework for understanding different means of category learning (Markman \& Ross, 2003). Later performance is usually influenced by the processing required by the task, not simply by what information is presented together. Considering all these reasons, it is highly unlikely that these results were due to learners simply learning what they saw.

Inherent differences between classification and inference

Under the assumption that labels and features are psychologically distinct, there are two critical interrelated differences between classification and inference: whether a label or feature is being inferred, and whether a label or a feature is already known. Both may contribute to performance differences between the tasks.

Inferring a label may lead learners to attend to different kinds of information, relative to inferring a feature. Category labels are arbitrary names referring to classes of objects, while features are meaningful parts of an object (Markman \& Ross, 2003). Because an item's features are

\footnotetext{
${ }^{9}$ Exp. 1: Inference learners' performance was nonsignificantly higher for features inferred during learning $(M=.73, S D=.23)$ than for those that were not inferred during learning $(M=.60, S D=.38), t(11)=$ $1.38, p>.05$.

Exp. 2: Inference learners' performance was better for features inferred during learning $(M=.88, S D=.19)$ than for those that were not inferred during learning $(M=.77, S D=.27), t(15)=2.61, s<.05$.
} 
likely to relate in meaningful ways to the feature in question, inference learners pay more attention to the item's other features than do classification learners, in an attempt to fit the feature being inferred in with other features presented (e.g., Erickson et al., 2005; Rehder \& Ross, 2001).

The information provided may also impact the learner's strategy. When classifying an item, the learner must consider all possible categories and determine which category this item is most likely to belong to. Alternatively, when inferring a feature of an object from an already specified category, one need only to think about that one category, regardless of the number of categories being learned (Chin-Parker \& Ross, 2004; Yamauchi \& Markman, 1998). This allows the learner to focus on what that particular category is like, independent of any other categories. In the majority of previous studies comparing classification and inference, only two categories were learned at once, so this difference was not as critical as in the present experiments, where six different categories were learned at once.

\section{Classification and inference outside the laboratory}

In their real-world forms, classification and inference are different from each other in a variety of ways. Here we examine some of the differences outside the laboratory and speculate on their implications. We focus on the variety of queries and the interrelatedness of features in real-world items.

First, although we queried only one feature in the inference condition in order to contrast theoretical explanations, inferences are usually made about a variety of properties, while classifications, by definition, ask for the category label. One cannot easily predict which properties of objects will need to be inferred, and this uncertainty would likely lead to attention to many of the features of a category (see, e.g., Rehder et al., 2009). So, while anticipatory learning cannot explain the performance differences here, it may contribute to some learning in the real world. Queried features are learned about more than nonqueried features (e.g., A. L. Anderson et al., 2002), so querying multiple features for inference is likely to lead to good learning about a variety of features.

Second, the interrelatedness of features in real-world items differentially influences the two tasks. In this research, as well as in other work that has contrasted classification and inference using artificial stimuli, the values of the experimenter-controlled features (in this case, the verbal features-e.g., finches eat nuts) were arbitrarily assigned with respect to the category and the other features. For real-world categories, a category's features are usually correlated. For example, inferring whether or not a dog will bite will be correlated with whether or not the dog has bared its teeth and whether or not it is growling. Inference learners are more likely to learn about these within-category correlations than classification learners, whether the correlations are meaningful (Erickson et al., 2005) or not (Chin-Parker \& Ross, 2002). Inference learners may actively look for correlations between features because they expect them to be there and to be potentially useful for making a future inference. Thus, experimental implementations that have tried to contrast these two tasks may reduce common differences between them that have important implications for their use in more real-world settings.

\section{Category learning and use}

These experiments show that two primary functions of categories, classification and inference, lead to differences in category knowledge during learning. This result may be viewed as part of a more general enterprise to understand the richness and flexibility of conceptual representations and how they are learned. There are many other tasks that require categories, and for these tasks, too, what is learned is likely to be related to how we use them. Although the focus of category learning has been on classification (an admittedly crucial function of categories), classification may differ in important ways from other category-learning situations to which the category knowledge is applied (often following classification), such as inference, problem solving, understanding, or constructing explanations (Markman \& Ross, 2003). Not only do we need to broaden the investigation to include other categorylearning tasks, we also need to consider that some of the flexibility of conceptual representations may arise from the multiple types of interactions with category members during learning.

\section{References}

Anderson, J. R. (1991). The adaptive nature of human categorization. Psychological Review, 98, 409-429.

Anderson, A. L., Ross, B. H., \& Chin-Parker, S. (2002). A further investigation of category learning by inference. Memory \& Cognition, 30, 119-128.

Chin-Parker, S., \& Ross, B. H. (2002). The effect of category learning on sensitivity to within-category correlations. Memory \& Cognition, 30, 353-362.

Chin-Parker, S., \& Ross, B. H. (2004). Diagnosticity and prototypicality in category learning: A comparison of inference learning and classification learning. Journal of Experimental Psychology. Learning, Memory, and Cognition, 30, 216-226.

Erickson, J. E., Chin-Parker, S., \& Ross, B. H. (2005). Inference and classification learning of abstract coherent categories. Journal of Experimental Psychology. Learning, Memory, and Cognition, 31, 86-99. 
Gelman, S. A., \& Heyman, G. D. (1999). Carrot-eaters and creaturebelievers: The effects of lexicalization on children's inferences about social categories. Psychological Science, 10, 489-493.

Gelman, S. A., \& Markman, E. M. (1986). Categories and induction in young children. Cognition, 23, 183-209.

Jacoby, L. L., Wahlheim, C. N., \& Coane, J. H. (2010). Retrieval practice enhances natural category learning: Effects on accuracy and on metacognition at the levels of items and categories. Journal of Experimental Psychology: Learning, Memory, \& Cognition, 36, 1441-1451.

Johansen, M. K., \& Kruschke, J. K. (2005). Category representation for classification and feature inference. Journal of Experimental Psychology. Learning, Memory, and Cognition, 31, 1433-1458.

Kruschke, J. K. (1992). ALCOVE: An exemplar-based connectionist model of category learning. Psychological Review, 99, 22-44.

Markman, A. B., \& Ross, B. H. (2003). Category use and category learning. Psychological Bulletin, 129, 592-613.

Murphy, G. L. (2002). The big book of concepts. Cambridge, MA: MIT Press.

Nosofsky, R. M. (1984). Choice, similarity, and the context theory of classification. Journal of Experimental Psychology. Learning, Memory, and Cognition, 10, 104-114.

Paivio, A., Rogers, T. B., \& Smythe, P. C. (1968). Why are pictures easier to recall than words? Psychonomic Science, 11, 1-2.

Rehder, B., Colner, R. M., \& Hoffman, A. B. (2009). Feature inference learning and eyetracking. Journal of Memory and Language, 60, 394-419.
Rehder, B., \& Hoffman, A. B. (2005). Eyetracking and selective attention in category learning. Cognitive Psychology, 51, 1-41.

Rehder, B., \& Ross, B. H. (2001). Abstract coherent categories. Journal of Experimental Psychology. Learning, Memory, and Cognition, 27, 1261-1275.

Rosch, E., \& Mervis, C. B. (1975). Family resemblances: Studies in the internal structure of categories. Cognitive Psychology, 7, 1573-1605.

Sakamoto, Y., \& Love, B. C. (in press). Learning and retention through predictive inference and classification. Journal of Experimental Psychology: Applied. doi:10.1037/a0021610.

Shepard, R. N. (1967). Recognition memory for words, sentences, and pictures. Journal of Verbal Learning and Verbal Behavior, 6, 156-163.

Yamauchi, T., \& Markman, A. B. (1998). Category learning by inference and classification. Journal of Memory and Language, $39,124-148$.

We thank Chris Wahlheim and Larry Jacoby for generously providing the bird pictures from their Bird Learning project, which were used in both experiments; the Ross lab meeting for their valuable feedback; and Robert Molitor for collecting and organizing the data. We also thank the anonymous reviewers for their comments on an earlier draft. 